\title{
Implications of the similarity principle relating creep and attenuation in finely grained solids
}

\author{
S.J.S. Morris ${ }^{\mathrm{a}, *}$, Ian Jackson ${ }^{\mathrm{b}}$ \\ a Department of Mechanical Engineering, University of California, Berkeley 94720, United States \\ ${ }^{\mathrm{b}}$ Research School of Earth Sciences, Australian National University, Canberra, ACT 0200, Australia
}

\section{A R T I C L E I N F O}

\section{Article history:}

Received 3 June 2008

Accepted 15 September 2008

\section{Keywords:}

Grain boundary sliding

Mechanical spectroscopy

Grain boundaries

Dimensional analysis

Similitude

\begin{abstract}
A B S T R A C T
The Raj-Ashby model of diffusionally accommodated grain-boundary sliding implies the existence of similitude in the mechanical loss spectrum of finely grained solids: the quality factor $Q$ of a shear wave propagating with angular frequency $\omega$ is related to the viscosity $\eta$ for steady-state Coble creep and the (unrelaxed) rigidity $\mu$ through a dimensionless relation of the form $Q=F(\omega \eta / \mu)$. Using existing measurements of $\eta$ and $Q$ obtained respectively from uniaxial creep tests, and forced torsional oscillation tests, we test that prediction. Though the similarity principle appears to hold, definitive proof will require further measurements of $Q$ and $\eta$ over a larger range of grain sizes. With that proviso, we conclude that the Raj-Ashby model includes the basic physical mechanisms acting in the experiments underlying two existing data sets on attenuation in the geological material olivine.
\end{abstract}

(C) 2009 Elsevier B.V. All rights reserved.

\section{Introduction}

The Raj-Ashby model of diffusionally accommodated grainboundary sliding implies that there is a relationship between the mechanical loss spectrum of a material deforming by diffusion creep, and its steady-state creep properties [1-3]. Specifically, the quality factor $Q$ of a shear wave propagating through the material with angular frequency $\omega$ is related to the viscosity $\eta$ for steady-state diffusion creep and the (unrelaxed) rigidity $\mu$ through a dimensionless relation of the form

$Q=F(\omega \tau) ; \quad \tau=\frac{\eta}{\mu}$

is the Maxwell relaxation time. According to Eq. (1), measurements of $Q$ made at different temperatures on samples having different grain sizes and activation energies for creep will define a curve when $Q$ is plotted against $\omega \tau$. In the geophysical context, if taken literally, Eq. (1) would imply that, once the form of the dimensionless function $F$ is found experimentally, the distribution within the mantle of the Maxwell timescale $\eta / \mu \sim 10$ Gs could be constrained by studying attenuation of seismic waves propagating through earth's mantle at periods $\sim 1 \mathrm{~s}$ [4].

The similarity principle would fail if any other physical mechanism influenced the value of $Q$. In that case, additional dimensionless parameters should be included in formulating Eq.

\footnotetext{
* Corresponding author.

E-mail address: morris@me.berkeley.edu (S.J.S. Morris).
}

(1). Measurements of $Q$ would then define a surface in an appropriate parameter space, rather than a curve in the $Q-\omega \tau$ plane. Unless the experiment were designed to fix the new parameters, the data would show scatter when plotted in the $Q-\omega \tau$ plane.

In the context of laboratory experiments on geological materials, two additional effects have, in fact, been proposed to explain discrepancies between the data and simplified analyses of the Raj-Ashby model: they are the effect of a boundary viscosity [3,5], and the possibility that some threshold phenomenon might be important at the low stresses ( $<1 \mathrm{MPa}$ ) occurring in the experiments [3]. If such effects were important, they would further complicate the problem of extrapolating experiments to mantle conditions.

The similarity principle has, however, been verified using the results of torsional oscillation tests and torsional creep tests on a finely grained polycrystalline geological material (olivine) [1,2,6,5]. Specifically, measurements of $Q$ made at different temperatures on samples having different grain sizes and activation energies for creep have been shown to define a curve when $Q$ is plotted against $\omega \tau[2,3]$. But if that proof of similitude withstands scrutiny, any discrepancies between experiment and theory would have to interpreted within the context of the Raj-Ashby model: namely the continuum theory of diffusionally accommodated sliding of elastic grains along grain boundaries on which the shear stress is zero. One would then seek to explain discrepancies between theory and experiment within a well-defined framework, rather than speculating that new physics might be required.

We have therefore revisited the proof of the similarity principle, Eq. (1). Cooper and coworkers tested the principle using values of $\eta$ using torsional creep tests that are in effect the low-frequency limit 
Table 1

Values $a, b$ obtained by fitting data from uniaxial creep tests [7] to Eq. (2).

\begin{tabular}{lll}
\hline$T(\mathrm{~K})$ & $a(\mu \mathrm{m})^{3}(\mathrm{MPa} \mathrm{s})^{-1}$ & $b(\mathrm{MPa})^{-2} \mathrm{~s}^{-1}$ \\
\hline 1423 & $2.0098 \times 10^{-7}$ & $3.2774 \times 10^{-12}$ \\
1458 & $2.8545 \times 10^{-7}$ & $1.9853 \times 10^{-11}$ \\
1509 & $4.5705 \times 10^{-7}$ & $1.4983 \times 10^{-10}$ \\
1523 & $1.4374 \times 10^{-6}$ & $2.4310 \times 10^{-10}$ \\
1573 & $4.4546 \times 10^{-6}$ & $2.8057 \times 10^{-10}$ \\
1583 & $5.8735 \times 10^{-6}$ & $5.8188 \times 10^{-10}$ \\
1623 & $1.3646 \times 10^{-5}$ & $1.0469 \times 10^{-9}$ \\
1633 & $1.9167 \times 10^{-5}$ & $1.2843 \times 10^{-9}$ \\
\hline
\end{tabular}

of the torsional oscillation tests used to measure $Q$; those values of $\eta$ were checked against just 4 uniaxial compression tests on a single sample. Here, we test the principle using values of $\eta$ obtained from a completely independent set of uniaxial creep tests [7]. Those tests were made at temperatures between 1400 and $1600 \mathrm{~K}$ on fully synthetic (sol-gel) samples with grain sizes in the range 3-6 $\mu \mathrm{m}$.

\section{Steady-state viscosity from uniaxial creep tests}

Because the tests were performed at stress differences spanning the wide range 14-211 MPa, the measured axial strain-rate $\dot{e}$ proves to be a weakly nonlinear function of the difference $\sigma$ between the maximum and minimum principle stresses. To obtain viscosities from those tests, we fitted the relation

$\dot{e}=\frac{a(T) \sigma}{d^{3}}+b(T) \sigma^{2}$

to the data. In the first (linear) term, the dependence on the cube of grain size $d$ is suggested initially by the fit given in Ref. [7]. (As a check, we also tried fitting the $d^{2}$ dependence that would correspond to creep by volume diffusion, but the resulting fit is much worse: values of $b$ in particular were badly scattered.) Using (2), a good fit usually required $\sigma$ to be less than $\sim 100 \mathrm{MPa}$, but in some cases data corresponding to stresses up to $200 \mathrm{MPa}$ could be used.

Table 1 shows the values of $a$ and $b$ thus found. Using the method of least squares to fit the relation between $\ln a$ and $1 / T$ with a line, we obtained the expression

$\ln a=21.569-\frac{53,330}{T}$;

$\ln b=17.329-\frac{61,280}{T}$,

similarly.

To demonstrate the fit, we introduce a dimensionless strain rate $\dot{e}_{*}$ and stress $\sigma_{*}$, defined as follows:

$\dot{e}_{*}=\frac{\dot{e} d^{6} b}{a^{2}}, \quad \sigma_{*}=\frac{b d^{3} \sigma}{a}$

According to Eq. (2), when graphed using those dimensionless variables, measurements of dimensional strain rate $\dot{e}$ made for differing values of $d, T$ and dimensional stress $\sigma$ should lie on the parabola

$\dot{e}_{*}=\sigma_{*}+\sigma_{*}^{2}$.

Fig. 1 shows the test of that prediction. Because the scale is linear rather than logarithmic, the linearity of the flow law near the origin is evident. To emphasize that linearity, in Fig. 1 we show only data for which $\sigma_{*} \leq 1$; when data for larger stresses are included, they show that the relation between $\dot{e}_{*}$ and $\sigma_{*}$ departs from the parabolic fit at a temperature-specific value of $\sigma_{*}$. Of course, Eq. (2) represents the first 2 terms in the Taylor expansion of the relation $\dot{e}(\sigma)$ about $\sigma=0$, and at higher stresses, the next (cubic) term in that expansion becomes important. At first, we assumed the parabolic term in that Taylor expansion to be zero, and used a cubic expression rather than Eq. (2). Though coefficients corresponding to $a$ and $b$ could then be

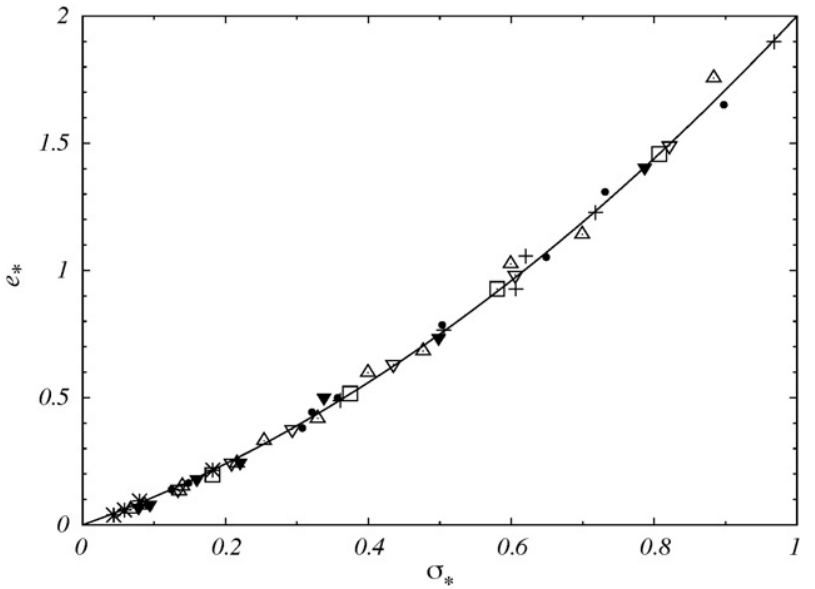

Fig. 1. Results of uniaxial compression tests [7] collapsed to emphasize the linearity of the relation between strain rate and stress near the origin. Symbols: $\left({ }^{*}\right) 1423 \mathrm{~K}$; $(+) 1458 \mathrm{~K} ;(\triangle) 1523 \mathrm{~K} ;(\bullet) 1573 \mathrm{~K} ;($ square) $1583 \mathrm{~K} ;(\nabla) 1623 \mathrm{~K} ;(\boldsymbol{\nabla}) 1633 \mathrm{~K}$. No data for $T=1509 \mathrm{~K}$ fall within the range of $\sigma_{*}$ included here. Solid curve, Eq. (5).

found with fair accuracy, that method was inconsistent because the corresponding dimensionless data defined a parabola.

The viscosity $\eta$ is given by the relation $\eta=(\mathrm{MPas})=\frac{1}{3} d^{3} / a$, where we note the numerical factor of $1 / 3$ appropriate to the uniaxial stress state. Substituting for $a$ from Eq. (3), we obtain

$\eta=1.43 \times 10^{-16} d^{3} \mathrm{e}^{53,330 / T}$.

In Eq. (6), $d$ and $\eta$ are expressed respectively in units $\mu \mathrm{m}$, and TPa s.

Fig. 2 shows viscosities so obtained. Heavy solid curves bound, approximately, the region within which Faul and Jackson took measurements. The other curves show the extrapolation of those data to the values of $d$ and $T$ occurring in the mechanical loss experiments discussed in Section 3. Two sets of lighter curves show different fits to the data: broken curves show the new fit Eq. (6), and light solid curves show the relation

$\eta=6.28 \times 10^{-18} d^{3} \mathrm{e}^{58215 / T}$,

the units being the same as in Eq. (6). Eq. (7) was obtained from the power-law relation given as Eq. (3) of Ref. [7] by evaluating the effective viscosity of the power-law material at the lowest stress

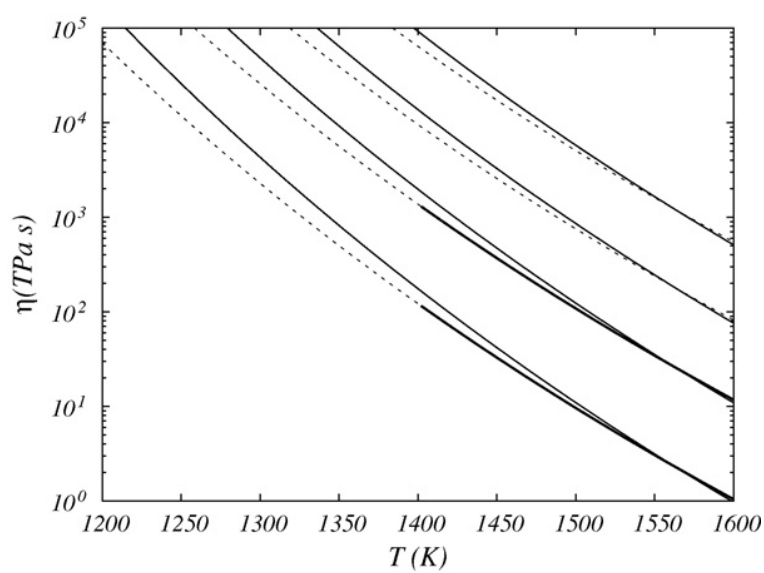

Fig. 2. Viscosity $\eta(T, d)$ of synthetic olivine samples obtained from uniaxial compression tests [7]. Heavy solid curves, Eq. (6), graphed for the approximate range of conditions within which measurements were made. Broken curves and light solid curves show the extrapolation of data using respectively Eqs. (6) and (7): from top to bottom $d=2.9,6.5,12.4$ and $23.4 \mu \mathrm{m}$. See Ref. [7] for comparison with the results of previous uniaxial compression. 
(14 MPa) used in those experiments. We note that although the pre-exponential factor in Eq. (7) is smaller than that in Eq. (6), the larger activation energy compensates for that difference, at least for the conditions covered in the figure. Because the figure shows that Eqs. (6) and (7) give comparable fits to the data, the mathematics of the extrapolation needed for our test of Eq. (1) are not at issue. Extrapolation may, however, be invalidated because the deformation mechanism could depend on grain size. Depending on the activation energies, volume diffusion might contribute increasingly to the creep rate. Unfortunately, for volume diffusion creep in olivine, we could only find estimates for the activation energy [8]: there appear to have been no measurements.

\section{Mechanical loss experiments}

Fig. 3 shows two independent sets of experimental results for forced torsional oscillations of fine-grained olivine samples [2,5]: we call these respectively the Bunton and the ANU data. All data are plotted for the value $\mu=65$ GPa quoted by Bunton. The Bunton data are for reconstituted olivine (Balsam Gap dunite) powders; all those data have been published already as a graph of $Q^{-1}$ against $\omega \eta / \mu[3]$.

For this paper, the Bunton data were plotted using values of $\eta$ which were measured by him using torsional microcreep experiments, and assumed to be steady-state values. ANU data for 2.9 and $12.4 \mu \mathrm{m}$ are for synthetic samples, and the remaining ANU data is for reconstituted San Carlos olivine. The ANU data were plotted using the value of $\eta$ obtained from the uniaxial compression tests, as summarized by Eq. (6) above. Because the data underlying that formula were collected for grain sizes less than $6 \mu \mathrm{m}$, extrapolation of $\eta$ over a factor of $2-25$ in $d$ is involved. Though the ANU loss experiments were also accompanied by microcreep tests, those data are not discussed here because, unlike the loss data, the microcreep data have not yet been recalibrated to account for a recently recognized interfacial contribution to the measured strain.

We note the following: (i) The Bunton data are, as noted previously [3], consistent with Eq. (1) because measurements for various values of $d$ and $T$ all collapse onto a single curve. Cooper [3] interprets the points near $\omega \eta / \mu=10^{2}$ to imply a flattening of that curve, and he suggests that flattening is an effect of a boundary viscosity. We doubt that is so, for the following reason.

Ashby [10] reviews theoretical arguments and experimental evidence showing that grain boundaries can, under certain condi-

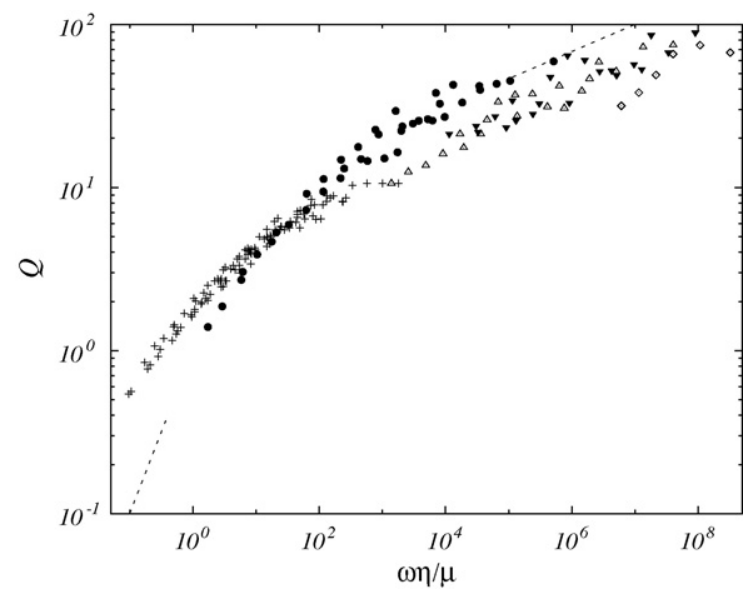

Fig. 3. Measured $Q$-values as a function of angular frequency $\omega$ in Maxwell units $\eta / \mu$. Symbol (+), Bunton [2,2] all data. Other symbols: all ANU data as recalibrated using 'double foil' calibration [9] for temperatures of $1273-1473 \mathrm{~K}$ in steps of $50 \mathrm{~K}$ for the following grain sizes in $\mu \mathrm{m}$ : $(\bullet) 2.9 ;(\triangle) 12.4 ;(\nabla) 23.4$; and $(\diamond)$ 165.1. Broken guidelines have slopes of $1 / 6,1$; their location is arbitrary. tions, resist shearing. According to theory, that effect introduces an additional dimensional parameter $\eta^{\prime} / \ell$, where $\eta^{\prime}$ is the so-called boundary viscosity and the length $\ell$ is of order the grain boundary thickness. From that parameter $\eta^{\prime} / \ell$, the grain size $d$ and the creep viscosity $\eta$, a second dimensionless parameter $\mathcal{M}=\eta^{\prime} d / \eta \ell$ can be constructed. Jackson et al. [5] use the Ashby theory to argue that the parameter $\mathcal{M} \propto(\ell / d)^{2}$. Consequently, if $Q$ were sensitive to the value of $\mathcal{M}$, there could not be a unique functional relationship between $Q$ and $\omega \tau$. (Because $\tau$ depends on grain size through $\eta$, in an experiment one could double the value of $\omega$, but keep $\omega \tau$ fixed by appropriately adjusting the grain size. According to the similarity principle Eq. (1), those two cases should result in the same $Q$. If, however, the second parameter $\mathcal{M}$ were significant, the change in grain size needed to fix $\omega \tau$ would cause $Q$ to vary. No unique relation between $Q$ and $\omega \tau$ would then be possible.) The absence of scatter from the Bunton data is inconsistent with the idea that a boundary viscosity is important, at least within the range $\omega \eta / \mu<100$. Instead it suggests that the parameter $\mathcal{M}$ is small so that the timescale based on the boundary viscosity is small compared with the diffusion timescale. In that case, the boundary viscosity would only become important at very high frequencies. We note that the absence of scatter from the steady-state creep data in Fig. 1 implies that in those experiments, the effect of the boundary viscosity is also negligibly small.

(ii) The ANU data appear to be more scattered, but closer scrutiny shows that most of what appears to be scatter is in fact due to a systematic offset in $\omega \eta / \mu$ between the curve for $d=2.9 \mu \mathrm{m}$, and data for all other grain sizes. That offset would be explained if the actual viscosity for those larger grain sizes is about one-tenth the value predicted by Eq. (6). That the Bunton data collapse onto a curve, but the ANU data are offset, suggests that neither Eq. (6) nor (7) is reliable at large grain sizes. Clearly, it would be useful to have the microcreep data for the ANU experiments. As noted in Refs. [5,3], both sets of mechanical loss data are more nearly consistent with a scaling in which the Maxwell time $\tau \propto d^{2}$, rather than the cubic scaling suggested by Eq. (6). Lastly, we do not believe the offset is an effect of boundary viscosity: if the parameter $\mathcal{M}$ were becoming significant, one would expect a gradual splitting of the curves with increasing $\omega \eta / \mu$, rather the observed systematic offset. However, this distinction may be difficult to draw with confidence amongst the inevitably more scattered, higher $Q$ values of the more coarsely grained materials.

(iii) In the figure, the broken line for $\omega \eta / \mu<0.5$ shows that neither data set approaches the low-frequency asymptote $Q \propto \omega$. Because Fig. 1 shows that a linear flow law is observable in uniaxial creep experiments, Fig. 3 simply reflects the inaccessibility of lower values of $\omega \eta / \mu$ in forced oscillation experiments. Attaining such values would require the suppression of grain growth in materials with $d<3-4 \mu \mathrm{m}$ at temperatures exceeding 1500-1600 K.

(iv) The broken guideline for $\omega \eta / \mu>10^{5}$ indicates that the slope of the $Q-\omega \eta / \mu$ relation decreases with increasing $\omega$. According to small-slope analysis [11] of the Raj-Ashby model for zero boundary viscosity, the dependence of $Q$ on $\omega \tau$ at high frequency is determined by the behaviour near corners, and physical reasoning based on an existing local analysis [12] suggests that is likely to be the case even for the more complex geometries occurring in polycrystals. Consistent with the observations in Fig. 3, those arguments predict a weakening dependence of $Q$ on $\omega \tau$ for $\omega \tau \gg 1$.

\section{Conclusion}

Spherically averaged seismic models of earth's mantle show that for shear waves $Q$ ranges from $\sim 100$ to $\sim 500$. Fig. 3 shows that even with the experimental advances made over the last decade, the asymptotic behaviour of $Q$ is not well constrained. However the experiments suggest that the effect of stress concentrations at 
grain edges are likely to control that asymptotic behaviour, at least in experiments where the dislocation density is minimal. The effect of those stress concentrations is now being addressed numerically. Though the similarity principle Eq. (1) is well-known, its implications seem not to have been understood. We believe that the present paper describes the first attempt to use similitude to argue that the Raj-Ashby model of diffusionally-accommodated grain boundary sliding includes all the physical mechanisms operating in a particular set of experiments. By using similitude to exclude suggestions that other physics should be incorporated into the Raj-Ashby model, we leave the way open to further detailed study of that model in its canonical form. Though existing data appear to support our approach, a thorough test of similitude in this context will require better data on the dissipation and steady-state viscosity of more coarsely grained samples.

\section{Acknowledgements}

We are grateful to Joseph Bunton and Reid Cooper for permission to use data from Ref. [2].

\section{References}

[1] T.T. Gribb, R.F. Cooper, J. Geophys. Res. 103 (1998) 27267-27297.

[2] J.H. Bunton, The impact of grain size on the shear creep and attenuation behaviour of polycrystalline olivine, MS thesis, University of WisconsinMadison, 2001.

[3] R.F. Cooper, in: S.-I. Karato, H.R. Wenk (Eds.), Rev. Geochem. Miner. 51 (2002) 253-290.

[4] U.H. Faul, I. Jackson, Earth Planet. Sci. Lett. 234 (2005), 119-134.

[5] I. Jackson, J.D. Fitz Gerald, U.H. Faul, B.H. Tan, J. Geophys. Res. 107 (B12) (2002), 2360, doi:10.1029/2001JB001225.

[6] B.H. Tan, I. Jackson, J.D. Fitz Gerald, Phys. Chem. Miner. 28 (2001) 641-664

[7] U.H. Faul, I. Jackson, J. Geophys. Res. 112 (2007), B04204, doi:10.1029/ 2006JB004586.

[8] B. Evans, D. L. Kohlstedt, in: T.J. Ahrens (Ed.), Global Earth Physics: A Handbook of Physical Constants, AGU Reference Shelf 3, American Geophysical Union, Washington DC, 1995.

[9] I. Jackson, A. Barnhoorn, Y. Aizawa, C. Saint, Phys. Earth Planet. Interiors 172 (2009) 104-115.

[10] M.F. Ashby, Surf. Sci. 31 (1972) 498-542.

[11] S.J.S. Morris, I. Jackson, J. Mech. Phys. Solids 57 (2009) 744-761.

[12] C.R. Picu, V. Gupta, Int. J. Solids Struct. 33 (11) (1996) 1535-1541. 